\title{
Identité queer: absence et négation de soi dans Folle de Nelly Arcan
}

\author{
Queer Identity: Absence and Self \\ Denial in Folle of Nelly Arcan
}

\author{
NADIA BouHadID \\ Université Batna2 \\ bouhadid.nadia@yahoo.fr
}

\begin{abstract}
Folle of the Canadian writer Nelly Arcan is the space of an attempt at self-reconstruction through an exposure of an identity antagonism generated by a complex relationship maintained with the Other. The exploration of the intimate life is then expressed as a process of recognition and self-denial; time, space and the body are then rationalized by a very stereotyped speech. Our study aims to explain the relationship between this self-reconstruction within an identity antagonism and autofictional writing, notably thanks to the concept of queer identity. Our approach will be based on a descriptive and analytical method which is part of an interdisciplinary approach drawing mainly on philosophy, psychoanalysis and studies on the issue of identity and interculturality.
\end{abstract}

\section{Key-words}

queer identity, spaciousness, autofiction.

\begin{abstract}
Resumen
Folle por la escritora canadiense Nelly Arcan es el espacio de un intento de auto reconstrucción a través de la exposición de un antagonismo de identidad generado por una relación compleja mantenida con el Otro. La exploración de la vida íntima se expresa entonces como un proceso de reconocimiento y abnegación; El tiempo, el espacio y el cuerpo se racionalizan mediante un discurso muy estereotipado. Nuestro estudio pretende explicar la relación entre esta auto-reconstrucción dentro de un antagonismo de identidad y la escritura autoficcional, en particular gracias al concepto de identidad queer. Nuestro enfoque se basará en un método descriptivo y analítico que forma parte de un enfoque interdisciplinario que se basa principalmente en la filosofía, el psicoanálisis y los estudios sobre el tema de la identidad y la interculturalidad.
\end{abstract}

\section{Palabras clave}

Identidad queer, amplitud, autoficción. 
J'en veux à la configuration des astres de rester imperturbable devant le trajet aléatoire des femmes dans la vie des hommes (Arcan, 2004: 85).

\section{Introduction}

Folle de l'écrivaine canadienne Nelly Arcan est son deuxième roman paru en 2004 aux éditions Seuil, un roman qui lui a valu le prix Femina. Nelly Arcan a été surtout connue par son premier roman Putain qui, en 2001, a fait une entrée remarquable dans la scène littéraire au Québec ainsi qu'en France, il s'agit d'une autofiction qui a pu lui décrocher une nomination pour les prix Médicis et Femina. Son œuvre aborde souvent des thèmes comme les représentations sociales et féminines, le suicide et la marchandisation du corps.

Folle est l'histoire de Nelly, ancienne prostituée et écrivaine, qui décide après une douloureuse rupture amoureuse d'écrire une lettre à son ex-amant, journaliste d'origine française, pour crier sa souffrance et sa détresse c'est alors qu'amour, mort et folie s'y mêlent. La folie traitée dans le texte est surtout celle d'une auteure-narratrice révoltée contre la sujétion de la femme dans une société réifiée où la femme est réduite à son paraître. Il s'agit aussi d'une révolte menée par l'auteure-narratrice contre les inégalités entre les deux sexes, et enfin une révolte contre la pornographie et la chosification du corps féminin. D'ailleurs, les titres de ses deux premiers romans, Putain et Folle, crient cette révolte comme elle l'affirme: Dans l'Histoire, "folle" et "putain" sont les deux mots qui ont marqué au fer rouge les femmes qui ne veulent pas se soumettre" (Arcan, citée in Nelly Arcan - La belle et le dragon 28 août 2004).

Notre présente étude tentera de mettre la lumière sur la construction d'une identité queer menée par la protagoniste de Folle au travers de l'écriture autofictionnelle. Nous tenterons d'examiner comment cette nouvelle identité, à la fois, sociale et politique est consciemment ou inconsciemment construite au sein du texte en mettant en exergue des identités antagonistes qui évoluent dans un espace de tensions.

Il serait intéressant de commencer notre étude par l'élucidation des frontières qui séparent les deux mouvements queer et féministe et montrer en quoi l'un serait l'aboutissement de l'autre et comment Arcan pourrait-elle aussi les faire coexister dans son œuvre.

\section{Identité queer et féminisme}

Le féminisme s'est toujours présenté comme un mouvement qui cherche à réhabiliter le statut et le rôle de la femme au sein de la société en rejetant tous les systèmes normatifs qui cherchaient à l'aliéner. Le combat mené par ce mouvement est axé sur la subversion des rapports de pouvoirs entre les deux sexes qui sont souvent au profit de l'homme, comme le dit à juste titre Simone de Beauvoir: "Les femmes ont toujours été définies par les hommes, 
relativement à eux" (Beauvoir, 1976: 35). Le féminisme encourage alors une construction de l'identité d'une femme-sujet en dehors des rôles sociaux qui lui sont assignés et en dehors du regard masculin et des rapports avec l'homme. Cette image d'une "nature" femme définie par une société patriarcale ne serait qu'un prétexte pour l'asservissement; Karen Offen explique justement que "le féminisme est le projet politique qui s'attaque à la domination masculine et à la subordination féminine" (Offen, 2009: 64). La force de cette identité féminine puiserait essentiellement dans le vécu et dans la subjectivité de la femme; ce sont donc ces deux éléments qui sont considérés comme facteurs de connaissance nécessaires pour pouvoir la guider vers son émancipation. Il s'agit dans ce sens d'un combat personnel et collectif puisque le féminisme "dans sa quête d'identité personnelle et collective, il donnait une dimension politique au personnel” (Pico, mai 2010).

Dans sa quête de libération de la femme, le féminisme a d'abord rejeté la maternité comme obligation sociale liée au "naturel féminin" en réclamant "la libre disposition de son corps" qui est la seule condition pour qu'elle puisse reprendre en main son destin. Il s'agit d'une liberté de procréation et non pas d'une négation de cette spécificité. Cependant, avec les progrès de la psychanalyse, le féminisme reprend la spécificité de la procréation comme trait de valorisation du genre féminin, élément lui procurant force et jouissance: "De tout temps ce qui fait la force, la jouissance des femmes: produire de la vie" (Groupe Psychanalyse et politique, 1979). C'est la glorification de cette différence des sexes qui a permis le retour à la définition traditionnelle de la maternité afin d'éviter tout risque de confusion avec le modèle masculin. Il faudrait noter que d'autres groupes féministes ont continué à lutter pour réfuter tout lien avec la maternité comme Christine Delphy (1970) qui, en se basant sur le modèle marxiste, verrait dans cette affirmation de spécificité, une occasion pour donner un prétexte au patriarcat à exploiter, tel le mode économique, la production féminine (l'appropriation des enfants par les pères).

Le féminisme se confronte ensuite avec le lesbianisme et se penche vers une voie radicale:

Au-delà des catégories de sexe, car le sujet désigné n'est pas une femme, ni économiquement, ni politiquement, ni idéologiquement. Car en effet, ce qui fait une femme, c'est la relation sociale, particulière à un homme, à laquelle les lesbiennes échappent en refusant d'être hétérosexuelles. Nous sommes transfuges à notre classe (Wittig, 1980: 43).

Le lesbianisme se posait ainsi comme un projet de libération "totale" en se détachant de l'hétérosexualité qui aliénait les femmes en étant un système basé sur une relation de dépendance des femmes aux hommes.

Les années quatre-vingts verront triompher une nouvelle conception du féminisme basée sur la distinction entre le sexe biologique et le gender: "l'ensemble des règles implicites ou explicites qui régissent les relations entre les hommes et les femmes" (Marzano, 
2013: 80). Le genre serait donc une catégorie capable de contourner les inégalités entre les deux sexes et redistribuer les rôles sociaux qui leur sont assignés. En 1990, Judith Butler définit le genre plutôt par sa "performance" et sa "performativité" (Butler, 2005): “C'est la performance qui produit rétroactivement l'illusion d'un noyau interne lié au genre, autrement dit l'illusion d'une essence ou d'une disposition masculine ou féminine" (Pico, op.cit ).

La praxis queer ${ }^{1}$, ayant eu un succès fulgurant pendant les années 1990 et faisant davantage parler d'elle de nos jours, remet en question la logique des mouvements qui l'ont précédée (féminisme, lesbianisme) qui étaient basés sur des distinctions identitaires relatives au sexe et au genre. En effet:

Les queer estiment les catégories d'opposition binaires (hommes/femmes, homos/hétéro) dépassées, voire essentialistes. Si le genre est performatif, il n'y a aucune raison de distinguer l'être et la performance, le genre est essentiellement autodéterminé; et chaque individu peut jouer à sa guise (Pico, op.cit .).

Le mouvement queer qui aspire à un effacement de frontières entre les deux sexesgenres s'opposerait-il au mouvement féministe? Nous rejoignons la réflexion d'Elsa Dorlin qui dans son ouvrage Sexe, genre et sexualités (2008) postule une nouvelle conception des deux mouvements, car selon elle le développement des théories féministes pourrait se lire comme une suite de "déplacements conceptuels: du sexe au genre, du genre à la sexualité, puis de la sexualité à la praxis queer" (Pico, op.cit .). Une telle lecture, avançant cette progression conceptuelle comme aboutissement nécessaire, ne fait pourtant pas l'unanimité au près des théoriciens féministes qui n'en voient qu'une réduction croissante du projet féministe.

Nous exploiterons l'identité queer dans Folle en visant l'aspect contestataire et autodéterminant dont elle se réclame, et voici la définition qui guidera notre réflexion:

Queer est un terme d'origine anglo-saxonne, réapproprié par les communautés LGBT de manière à en faire un symbole d'autodétermination et de libération plutôt qu'une insulte. Il fait référence à toute idée, pratique, personne ou identité allant à l'encontre des normes structurant le modèle social hétéronormatif. En ce sens, le terme connote une autoreprésentation contestataire. (Définitions sur la diversité sexuelle et de genre, 2016).

Les queer renvoient donc aux personnes qui refusent d'être étiquetées selon leur orientation sexuelle ou leur identité de genre et s'insurgent ainsi contre l'hétéronormativité. Il faudrait, cependant noter qu'en critique et théorie littéraires, "le queer dépasse alors la simple étude de l'homosexualité dans la littérature: il débusque le "pervers" dans les textes, il étudie les stratégies par lesquelles les œuvres subvertissent les catégorisations sexuelles et le système de genre" (Minne, 2009: 32).

1 “Altersexualité" et "allosexualité" sont souvent les traductions proposées pour le mot queer. 
Ce sont donc ces stratégies de subversion du genre que nous tenterons d'explorer dans Folle en y étudiant le fonctionnement et la fonction de la négativité et de l'anti-socialité; nous tenterons alors de démontrer que l'aliénation (auto-aliénation) et le défaitisme s'inscrivent plus dans la contestation que dans la soumission. Cette négativité/anti-socialité se manifesterait essentiellement par un refus de "productivité" sentimentale, sociale et existentielle.

\section{La maternité et le non futur}

La négativité se manifeste d'abord par le rejet de l'auteure-narratrice d'une vie future qui entraînera, chez elle, un refus de la maternité et de ses joies. En effet, nous découvrons dès les premières pages du roman une narratrice déterminée à se donner la mort la veille de ses trentaines, une date qu'elle s'est fixée dès son adolescence: “Le jour de mes quinze ans, j'ai pris la décision de me tuer le jour de mes trente ans" (Arcan, 2004:13-14). Une décision peu commune qui fait résonner son titre Folle, une folie qu'elle expérimente au quotidien en vivant tout en sachant que la date limite se rapproche de plus en plus, et que le futur lointain est une expérience hors limites; tout évènement est alors scruté à travers le crible de cette date. D'ailleurs, quand la narratrice rencontre le journaliste français qui deviendra son amant, sa mort proche est alors l'un des premiers obstacles qui se tient entre eux: "quand on s'est rencontré la première fois à Nova, j'allais avoir vingt-neuf ans sur le coup de minuit. Le problème entre nous était de mon côté, c'était la date de mon suicide fixée le jour de mes trente ans" (Ibid.: 13-14).

Le défaitisme qui s'empare de l'auteure-narratrice la poussant à penser au suicide, remonte à son enfance quand ses parents, à cause d'un mauvais diagnostic, s'attendaient à avoir un garçon: "[...] le médecin qui avait suivi ma mère pendant la grossesse lui avait annoncé un garçon, il lui avait même montré la présence d'une petite queue sur l'échographie" (Ibid.: 161). Cette anecdote racontée autour de son évènement de naissance a marqué l'esprit de l'auteure-narratrice qui développe un complexe face au manque du "phallus" dont parle Freud, un complexe qui la pousse à chercher à devenir un homme mais en vain: "il est possible que mes efforts pour être un homme ne me rendaient que plus femme encore" (Ibid.: 110). Ce manque s'accentue de plus en plus en constatant le pouvoir octroyé aux hommes au sein de sa communauté: "Oh mon Dieu que je déteste la force des hommes à ne pas être concernés, mon Dieu que j'aimerais être un homme pour ne pas avoir à dire ces choses-là" (Ibid.: 12).

L'auteure-narratrice pense alors que son destin était celui de ne pas exister car même devant les tarots de sa tante son avenir refusait de se dévoiler:

Quelque chose en moi n'a jamais été là. Je dis ça parce que ma tante n'a jamais pu voir mon futur dans ses tarots, elle n'a jamais pu me dire quoi que ce soit de mon avenir, même quand j'étais une enfant non ravagée par la puberté. Je suppose que pour certains le futur ne commence jamais ou seulement passé un certain âge. (Ibid.: 12). 
Elle n'est toutefois pas surprise par le mutisme des cartes puisque sachant qu'elle est déterminée à se donner la mort à la veille de sa trentaine, aucun avenir n'est alors envisageable pour elle: “Je sais aussi que mon existence la remettait en question, sans doute qu'elle déplorait que ses tarots ne sachent pas représenter le doute, l'inertie, ou le temps figé des gens qui attendent la mort" (Ibid.: 13). Le rapport à l'Autre chez la protagoniste de Folle est, à cet effet, influencé par le paramètre d'un futur impossible, la communication doit alors se faire dans une logique qu'elle seule, pourrait comprendre: "Depuis la loi du silence des tarots de ma tante sur mon existence, je marche à l'envers des autres" (Ibid.: 128).

La décision du suicide entraîne ainsi l'auteure-narratrice dans une non projection dans le futur et la transforme en un être asocial qui prend beaucoup de distance par rapport aux autres. La négativité acquiert alors le visage d'une asocialité car l'auteure-narratrice tout en ayant un statut d'auteure publiée et étant exposée souvent aux médias, elle se sent pourtant comme un être isolé, souffrant de l'angoisse du danger de l'Autre:

Ma folie te dépassait, elle te jetait par terre. Tu détestais ma façon de me déclarer faible et de parler des autres en termes de danger, tu disais que pour moi les autres rayonnaient trop et que je devais m'en protéger en les regardant de loin. D'ailleurs au Bily Kun, j'avais tendance à finir les soirées dans un coin, en moi il y avait un élan naturel de retrait, la génuflexion venait toute seule. (Ibid.: 142).

L'écriture ne lui permet pas, à contrario, d'être plus accessible et plus ouverte aux autres, elle accentue davantage son isolement comme elle le fait remarquer à son amant: “devenir écrivain était pour toi un vieux rêve et pour moi l'aboutissement de mon asocialité» (Ibid.: 170).

La crise existentielle de la narratrice autodiégétique affecte également son désir de maternité puisque même si elle tombe enceinte, elle décide de s'en débarrasser pour poursuivre son destin fatal. La grossesse a été d'abord utilisée par la narratrice comme un fragment relique ${ }^{2}$ afin de pouvoir faire le deuil d'une histoire d'amour dont elle n'arrivait pas à se détacher; il s'agit donc d'un acte de désespoir cherchant à garder l'autre absent bien présent en soi: 'En sentant venir ton départ, j'ai voulu faire quelque chose et j'ai fait un enfant, à ce moment il me restait quatre mois à vivre. Cet enfant je l'ai fait sans y croire vraiment, trop d'obstacles devaient être surmontés pour parvenir à une éclosion» (Arcan, 2004: 167-168). Elle est même allée jusqu'à simuler une permutation entre le fragment relique et la figure de l'absent comme elle l'explique: “je suppose aussi que depuis le début des temps, les femmes ont usé de cette capacité redoutable de rendre les pères et les bébés interchangeables, et c'est peut-être en vertu de cette capacité que bien souvent elles se désintéressent des pères une

2 "Fragment d'un corps disparu en lequel se recueille le souvenir de l'être dans sa totalité, la relique est objet sacralisé qui, à la faveur d'une véritable dérision du quotidien, interdit au disparu de séjourner désormais parmi les habitudes et lui assigne pour résidence quelques pauvres restes retenus de lui ou prélevés de son apparence", (Fedida, 1978: 76). Dans le cas de l'auteure-narratrice de Folle le fragment relique ne renvoie pas à un objet, mais il est représenté, dans un sens figuratif, par l'image du bébé. 
fois les bébés mis au monde" (Ibid.: 69). L'auteure-narratrice est ainsi incapable de faire le deuil puisqu'elle n'accepte pas la rupture, et comme c'est l'amant qui a mis fin à leur histoire amoureuse, elle décide alors de le devancer en ayant, cette fois-ci, un pouvoir sur lui puisque, par permutation avec le bébé, c'est lui qu'elle emprisonne dans ses entrailles: "Pour la première fois entre nous tu étais le plus petit, pour la première fois je te surplombais avec de mauvaises intentions. C'est moi qui désormais étais en mesure de voir le dessus de ta tête, certes tu ne pourrais pas t'échapper parce que de cette cage, c'est moi qui avais la clé" (Ibid.: 69).

Cette grossesse n'est pas seulement un acte de vengeance mais c'est aussi et surtout un cri de révolte contre le pouvoir de son amant, en tant que figure autoritaire; tomber enceinte serait à cet effet un droit légitime qu'elle le lui arrache:

\begin{abstract}
Quand on est rentré ce soir-là on s'est disputé, je voulais savoir si un jour, tu voudrais un enfant de moi, et toi tu voulais savoir si un jour, je n'aurais pas le culot de te faire un enfant dans le dos. Tu as dit que, pour les hommes de ta génération, le potentiel des femmes à négocier seules la reproduction était un cauchemar parce qu'il pouvait hypothéquer un début de carrière [...] ce soir-là je t'ai répondu que par égocentrisme les hommes transformaient des désirs légitimes en mauvaises intentions à leur égard, que les hommes avaient le don de se croire à l'origine du moindre battement de cils des femmes. (Ibid.: 64).
\end{abstract}

Tout en imposant son droit de tomber enceinte, Nelly réclame aussi son droit d'avortement; c'est surtout pour elle une manière de s'évider de la présence-absence de l'amant: "pour me faire avorter j'ai attendu la toute dernière minute légale, je suppose que ça a été une façon de te retenir le plus longtemps possible après ton départ” (Ibid.: 69) ou encore: “j’ai eu peur qu'en plus de ton nom, je lui donne ton prénom" (Ibid.: 70). Cette décision d'avortement est aussi encouragée par la nature de son ex-amant qui n'aimait pas les enfants: "un jour tu m'as dit qu'en manquant de gêne, les enfants te gênaient et que de ta vie, tu n'en avais jamais pris un sur tes genoux" (Ibid.: 74). L'avortement qui est dans un sens le fait de se débarrasser du fragment relique est surtout motivé par l'incapacité de ce fragment à aider l'auteure-narratrice à faire le deuil puisqu'il accentue beaucoup plus la présence de l'objet perdu (l'amant):

Pendant le troisième mois, j'ai beaucoup hésité. Dans l'ennui qu'était devenue ma vie depuis ton départ, je voulais créer du suspense, sans doute que je voulais me réserver une bonne surprise, qui sait si le temps passant le bébé ne me déchargerait pas de ton poids. Durant ces trois moi ce n'est jamais arrivé, en aucun moment tu t'es retiré de moi, c'est peut-être tout simplement parce que tu ne savais rien de ce qui était en train de m'arriver. (Ibid.: 69).

Le fragment relique dans ce cas de figure ne remplit pas sa fonction qui est celle d'aider le sujet souffrant à se détacher progressivement de l'objet perdu, mais il accentue 
davantage sa présence-absence qui torture l'auteure-narratrice. Cependant, selon Fédida "La relique tire son sens - à la fois sa nature et son pouvoir - du renversement possible d'une signification de l'objet en sa signification contraire" (op.cit:: 87). En effet, l'auteure-narratrice renverse le lien du fragment relique, représenté par le bébé, ayant un lien explicite avec le père (l'ex-amant) et le détourne vers elle en supposant qu'il pourrait lui ressembler et tout hériter d'elle: “puis finalement j'ai eu peur qu'il me ressemble, qu'il soit comme moi sans avenir, que lassé de lui-même il finisse par te chercher pour tomber sur ta grandeur démesurée capable de le renier" (Arcan, 2004: 70). Ce premier glissement de la représentation du fragment relique va encore être réorienté vers un sens différent: c'est l'enfant qui décide de s'auto-éliminer dans le ventre:

Puis j'ai eu peur qu'il ne ressemble à rien, qu'il ne soit déjà mort de chagrin avant terme, il paraît que les enfants se laissent mourir de faim dans le ventre des mères abandonnées et incapables de sortir de leur état de choc, qu'ils rompent avec elles toute communication en arrachant le cordon ombilical avec leurs propres mains (...) il parait que les enfants aiment leurs mères à ce point-là. (Ibid.: 70).

L'amour a donc motivé l'auteure-narratrice pour tomber enceinte en interprétant notamment un geste de son amant: "en me voyant pleurer, tu as posé la tête sur mon ventre et j'ai supposé que ce geste faisait de toi un père. Trois mois plus tard, j'étais enceinte de toi" (Ibid. : 65). La grossesse a toutefois remis en question son rapport avec la mort: "Peu après, un test de pharmacie a confirmé ma grossesse et les choses ont changé pour un temps, le moment de ma mort a été remis en question" (Ibid.: 113), ou encore: "Pendant ce troisième mois, j'ai d'abord pensé que le bébé et moi on pourrait se sauver mutuellement la vie, qu'en ayant tous les deux la corde au cou on établirait entre nous un pari d'endurance" (Ibid.: 69). Toutefois, le fragment relique n'a pas rempli sa fonction puisqu'il est censé rappeler “que le deuil, avant de se concevoir en un travail, protège l'endeuillé contre sa propre destruction" (Fedida, op. cit.: 98); en effet, la grossesse en tant que fragment relique n'a pas réussi à convaincre l'auteure-narratrice de changer d'avis et de reporter son suicide: “Ce n'est que le lendemain de l'avortement que ma décision est redevenue irrévocable et que j'ai commencé à t'écrire cette lettre" (Arcan, 2004: 113). L'auteure-narratrice a donc décidé de se débarrasser du fragment relique la reliant à son ex-amant, le deuil semble ainsi achevé et cela se révèle dans le changement de sentiments le jour de l'avortement: “Ce soir-là j'ai commencé à te haïr et jusqu'à ce jour cette haine est restée la même. La haine est une composante stable" (Ibid.: 80).

Cependant, faire le deuil ne veut pas dire avoir le soulagement car en écrivant cette lettre où l'auteure-narratrice avoue être tombée enceinte sans avoir le consentement de son amant, et avoir avorté pour l'oublier, ne l'a pas aidée à avoir la sérénité: “on dit souvent que l'aveu soulage. Pourtant jusqu'à ce jour (avortement) je ne l'ai pas senti en écrivant cette lettre, c'est peut-être parce qu'elle ne s'adresse pas vraiment à toi' (Ibid.: 69). Il s'agit chez l'auteure-narratrice surtout d'écrire sur l'impossibilité de faire le deuil: 
En fait, j'écris sur l'impossibilité de faire le deuil de ma condition d'unique et d'irremplaçable. La folie n'est pas loin, car la non-folie serait d'accepter sa place dans le monde. Entre immense orgueil et immense modestie, l'écriture demeure pour moi une forme très sophistiquée d'autoflagellation (Arcan, citée in Nelly Arcan -La belle et le dragon, 28 août 2004).

Arcan revient, une fois encore, sur sa conception de l'écriture qu'elle considère comme une manière de torture, de descente aux enfers afin d'arriver à une purgation qui n'est toutefois assurée qu'en combinant deux alternatives: folie et mort.

\section{Spacieux: espace de tensions et d'antagonismes}

Si chacun de nous dessinait son propre corps sous la dictée de son regard intérieur, on obtiendrait une belle galerie de monstres! (Millet, 2002: 189).

Afin de donner une dimension plus intense à la souffrance de la protagoniste de Folle, Arcan décide d'installer au sein de son texte un spacieux ${ }^{3}$, pour ainsi reprendre la terminologie de Derrida, capable de faire coexister les antagonismes et de les nourrir de tensions. Le spacieux déclenche une série d'oppositions qui finissent par se disloquer créant ainsi un espace d'espacement et de contradictions où la réconciliation n'est parfois qu'un projet utopique.

La première opposition qui se manifeste dans Folle est celle de la vie contre la mort. En effet, la narratrice décidée de se tuer la veille de ses trentaines, rencontre l'amour qui remet en question son rapport avec la mort: “[...] je t'ai aimé tout de suite sans réfléchir à ma fin programmée depuis le jour de mes quinze ans [...] Quand on s'est mieux connus, c'est devenu un problème" (Arcan, 2004: 7), car selon elle "le problème entre nous était de mon côté, c'était la date de mon suicide fixée le jour de mes trente ans" ( Ibid.: 14).

Nelly a vécu un tiraillement entre ces deux pôles ce qui n'a fait qu'accentuer son angoisse et sa dépression. Cependant, l'auteure-narratrice a pu apaiser ses tensions quand l'un des pôles d'opposition a cessé d'exister, celui de l'amour. En effet, quand son amant l'a quittée, la narratrice autodiégétique de Folle décide de mettre son plan de suicide à exécution, une intention qu'elle révèle justement vers la fin de son œuvre qu'elle conclut ainsi:

Cette lettre est mon cadavre, déjà, elle pourrit, elle exhale ses gaz. J'ai commencé à l'écrire au lendemain de mon avortement, il y a un mois.

Aujourd'hui ça fait exactement un an qu'on s'est rencontrés.

Demain, j'aurai trente ans. (Ibid., 205).

3 "Le spacieux déclenche une chaîne qui disloque les oppositions et propage la lettre, la déplie comme un hymen. Il forme un espace dépourvu de référent, toujours supplémentaire. La scène, quoique mentale, se joue dehors, hors de l'esprit, dans une marche qui ouvre une crise" (Derrida, 1972: 288). 
Anales de Filología Francesa, n. ${ }^{\circ}$ 27, 2019

IDENTITÉ QUEER: ABSENCE ET NÉGATION DE SOI DANS FolLE DE Nelly ARCAN

Si Arcan a choisi de programmer sa mort dès l'âge de quinze ans, pour des raisons qu'elle n'a pas révélées, après la rupture avec son amant, elle décide d'en parler:

Les raisons de mourir ont varié selon les hommes qui sont entrés dans ma vie. Le jour de mes trente ans comme date limite a été fixé depuis longtemps mais les motifs ont bougé $[\ldots]$ je mourrai parce que, pour être aimée des autres, il m'aurait fallu sourire. Je mourrai pour démontrer que le sourire est une façon de s'économiser comme le sommeil. [...] le sourire des gens tristes a toujours quelque chose de laborieux. (Ibid.: 144).

L'auteure-narratrice montre donc que le choix du suicide est, pour elle, une manière de donner une leçon aux autres, car ce sont les autres qui ont décidé que l'amour ne peut s'acquérir qu'avec la condition d'afficher son sourire, symbolisant sa joie. L'auteure-narratrice joue alors avec les oppositions joie/tristesse, spontanéité/labeur pour montrer que la notion de l'amour, chez elle, est basée sur un ressenti personnel soumis obligatoirement au crible du jugement des autres. L'accès à soi ne se fait alors qu'avec l'accord d'autrui, et être aimée suppose, par conséquent, manifester le comportement normé des amoureux et dessiner un grand sourire sur son visage. Arcan compare le sourire au sommeil, pour montrer que cette manifestation de joie n'est pas représentée de la même manière chez tout le monde et que sourire pourrait devenir, chez certains, une vraie torture et non pas une manifestation de plaisir. En effet, Lacan souligne qu'“au niveau de l'expérience des autres [...] le sujet a à reconnaître ses désirs. Et s'ils ne sont pas reconnus, ils sont comme tels interdits, et c'est là que commence en effet le refoulement" (Lacan, 1973: 42). Se donner la mort serait alors pour elle une manière de se révolter contre les normes sociales et contre le conformisme qui font de l'être humain un être conditionné et l'obligent à adopter des comportements qui ne sont pas nécessairement compatibles avec ce qu'il ressent, ou ce qu'il pense. L'absurdité de la vie, et la révolte contre le conformisme social sont ainsi au cœur de la réflexion dans Folle.

En outre, Arcan met en exergue d'autres oppositions qui sont essentiellement dynamisées par des stéréotypes très féministes. Dans Folle l'auteure-narratrice s'interpelle et s'évalue sans cesse à travers le regard de l'Autre. Elle commence d'abord par se chercher à travers le regard de son amant français, un journaliste venu à Montréal pour s'initier à 1 écriture. La différence d'appartenance historique et géographique rappelle de tout de suite à Nelly l'opposition colonisateur/colonisé, une opposition qui l'encourage surtout à tomber sous son charme: “Aujourd'hui je sais que je t'ai aimé à cause de ton accent de Français où s'entendait la race des poètes et des penseurs venus de l'autre côté du monde pour remplir nos écoles [...] cet accent qui te séparait de tout le monde, des Québécois comme des Français" (Ibid.: 8). Nelly explique alors que le contact avec son amant nourrit chez elle un sentiment contradictoire: d'une part, celui de l'admiration étant donné que l'accent de son amant renvoie à un héritage historique auquel elle s'identifie, et d' autre part, il lui rappelle sans cesse la situation du québécois colonisé et méprisé: 
Malgré ton accent de Français, tu parlais le québécois plus encore que les québécois, n'étant pas né ici tu ne portais pas la honte. D'être méprisé par la masse des anglais et objet de moquerie des "Français de France" comme on dit ici pour doubler la distance, pour laisser au Français la paternité du français, restait pour toi une abstraction. D'ailleurs à n'importe quel moment tu pouvais retourner dans ton pays d'origine, ça te faisait aimer le Québec. (Ibid.: 34-35).

Éprise de son amant, l'auteure-narratrice ne peut s'empêcher pourtant d'envier son statut d'étranger au Québec:

Tu parlais ma langue en sachant que tu ne connaîtrais jamais l'opprobre où vivent les colonisés, en sachant aussi que l'assimilation n'atteindrait jamais les couches profondes de ta personne, et que ton pays d'origine te protégerait à jamais du besoin d'être reconnu. (Ibid.: 35).

Perturbée par cette situation contradictoire, l'auteure-narratrice n'éprouve aucun plaisir, par exemple, quand son amant tente d'apprendre le québécois, mais elle ressent plutôt un sentiment de malaise: "Tu disais plotte, slut, slack, fun, pitoune, se crosser, tu disais t'en crisser, tu me renvoyais tous ces mots que j'avais mis des années à désapprendre, par exemple du disais avoir une blonde et non avoir une copine ou une petite amie" ( Ibid.: 35). Entendre du québécois prononcé par son amant, représente chez la protagoniste, une sorte de reflet de l'image de soi à travers la parole de l'autre et c'est pour cette raison qu'elle utilise l'expression "tu me renvoyais tous ces mots" pour exprimer cet effet-miroir. Nous constatons donc que la représentation de soi chez la narratrice est péjorative, entraînant de ce fait une négation de sa propre subjectivité et donc un rejet identitaire.

La dévalorisation de soi chez la narratrice se manifeste également quand elle aborde l'opposition brune et blonde. En effet, Nelly développe une sorte d'obsession en comparant sans cesse la blonde et la brune, notamment quand elle apprend que son amant n'a aimé, avant elle, que des brunes: “Avant moi tu n'avais connu que des brunes. [...] Mais le mystère de ton amour reste entier parce que je n'étais pas brune" (Ibid.: 20-21). Être blonde ne distingue pas seulement l'auteure-narratrice dans la vie amoureuse de son amant mais, selon elle, le qualificatif "blonde" affecte surtout l'imaginaire collectif québécois:

Personne ici ne m'a jamais expliqué pourquoi au Québec toutes les petites amies sont des blondes, sûrement qu'il y eût jadis une époque de suprématie des blondes sur les brunes comme celle des Blanches sur les Noires [...]. Je me demande si un jour au Québec les blondes se feront massivement teindre les cheveux en brun, je me demande aussi à quel moment les Québécois en auront assez de dire ma blonde pour désigner celle qu'ils aiment, à quel moment ils apercevront que les brunes sont bien souvent les plus belles. (Ibid.: 35).

C'est l'aspect social du stéréotype de l'opposition brune/blonde qui déstabilise la représentation de l'image de soi chez l'auteure-narratrice. La citation ci-dessous montre que la 
narratrice dévalorise sa propre image en tant que "blonde" en affirmant que la beauté est liée systématiquement aux brunes. Le discours de Nelly dévoile ainsi un complexe qu'elle a développé face aux brunes, un complexe qui est renforcé en raison du regard social représenté essentiellement par l'avis masculin: "une blonde même belle ne fait pas le poids devant un homme qui a besoin de la chaleur collectivement admise d'une brune" (Ibid.: 22).

La protagoniste de Folle semble donc totalement soumise aux stéréotypes du genre qui répertorient l'univers masculin/féminin en catégories stéréotypées: dominant/ dominé et désirant/ désiré. Étant blonde, ce caractère physique du corps féminin qui est généralement dévalorisé dans l'univers du désir masculin - phénomène causé par l'ampleur des stéréotypes-, perturbe la représentation de soi chez l'auteure-narratrice qui perd confiance en elle et devient obsessionnellement jalouse.

La protagoniste de Folle tente donc d'atteindre un idéal féminin validé par le regard masculin, et cela en remodelant son corps au moyen de la chirurgie esthétique au risque de ne plus se reconnaître: “[...] J'en ai assez de ses manœuvres de séduction de laboratoire qui ont trop souvent eu ma peau" (Ibid.: 22). Le discours stéréotypé et hystérique de l'auteure-narratrice dévoile une identité extrêmement fragile qui se cache derrière une aspiration à une image féminine exempte de tout manque, et donc ouvrant le droit à une validation symbolique, et c'est bien cela qu'exige le personnage Nelly de son amant.

Elle construit de ce fait son identité face à l'autre dans un mouvement de morcellement, c'est-à-dire que sa représentation de sa propre identité se projette dans le reflet de certains aspects de l'autre sélectionnés et intégrés à son imaginaire. Dans ce sens, Lacan écrit: “l'hystérique a toutes sortes de difficultés avec son imaginaire, ici représenté par l'image de l'autre, et [...] elle est susceptible d'y voir se produire des effets de morcelage, diverses désintégrations, qui sont ce qui lui sert dans son symptôme” (Lacan, 1998: 389).

Le discours stéréotypé de l'auteure-narratrice est ainsi un révélateur d'une identité sans repères, soumise à l'idéologie culturelle de son milieu et entachée de zones d'ombre d'un manque tant refoulé.

Le spacieux dans Folle représente d'abord une chaîne de tensions constantes entre la narration de l'objet et l'auto-narration du sujet. L'auteure-narratrice enchaîne des oppositions de nature différente qui s'accentuent au fur et à mesure de l'écriture dans un mouvement d'écriture hors de soi afin de saisir une image de soi compatible avec son imaginaire. Le spacieux témoigne d'un malaise qui accompagne notre auteure-narratrice dans sa découverte de soi.

Contrairement aux autobiographes qui aspirent à maîtriser l'espace par la transparence d'une parole chaleureuse et régénératrice, notre autofictionnaliste jouerait de l'excès du signifiant qui ouvrirait l'espace et le mettrait en mouvement dans un univers mental, hors de l'esprit comme l'écrit Derrida "le monde mental est déjà une scène, le dedans de la mens" (Derrida, 1972: 288). Le spacieux est à cet effet le mouvement dynamique de la fiction, il forme une scène régie par l'auto-analyse qui fouille au cœur d'une crise. 


\section{La sexualité et l'absence à soi}

Le désir qui ne connaît de réalité que lui-même [...] (Arcan, 2001: 13)

La sexualité au sein du couple arcanien est essentiellement basée sur le modèle stéréotypé homme actif/femme passive, c'est-à-dire que c'est l'homme qui prend l'initiative, agit et commande, alors que la femme ne fait que subir, coopérer et accepter les fantasmes de l'autre. Nelly le confirme en s'adressant à son amant: “c'est toujours toi qui prenais l'initiative, qui toujours choisissais le moment propice" (Arcan, 2004: 60) ou encore: "Quand tu voulais me faire prendre une direction, celle du lit par exemple, tu m'empoignais la nuque en exerçant une pression qui me poussait là où tu voulais" (Ibid.: 39). L'homme est donc le maître absolu de la relation sexuelle au sein du couple arcanien, et sa possession du "phallus" semble lui conférer l'essence même de l'existence (Cornaton, 1990: 41), tandis que "la femme n'a pas d'autorité spécifique: elle ne détient que celle concédée par l'homme dès lors qu'il exerce son pouvoir sexuel" (Ibid.: 40). En outre, toute relation sexuelle égalitaire devrait être basée sur un échange et un partage mutuel de plaisir car à travers "la possession du corps d'autrui, une personne prend contact avec l'être charnel de la personne qu'il désire et cherche à réaliser une union sexuelle, union ayant le but de permettre une connaissance et l'émergence d'une subjectivation mutuelle" (Marzano, 2002: 92). Or, au sein du couple arcanien, "le plaisir sexuel direct est réservé au mâle, ce qui renforce la dépendance féminine: les femmes ne peuvent s'épanouir sexuellement que par identification, par substitution à l'homme qui les possède" (Fireston, 1972: 189) et c'est justement à ce niveau-là que transparait une forme d'aliénation.

Le rôle passif de la narratrice est toujours très présent dans sa vie sexuelle, et même en tentant de prendre l'initiative, elle ne peut aller jusqu'au bout puisque consciemment ou inconsciemment, elle est freinée par le stéréotype de l'homme-maître: “[...] j’ai préféré mettre un doigt dans ton nombril pour te chatouiller. Je suppose que ça a été une façon de te passer le flambeau, de t'indiquer le chemin à prendre vers moi, c'est d'ailleurs devenu un classique entre nous, mon signal de départ" (Arcan, 2004: 60). L'expression "passer le flambeau" dévoile impartialement l'attitude démissionnaire et surtout passive de la narratrice, et donc son aliénation consentie à sa situation d'objet de désir, mais en ayant constamment l'amour comme motif: “[...] J'ai eu envie d'être salie et frappée. Pour la première fois il m'a semblé que mon amour t'en donnait le droit et même l'ordre, d'ailleurs si tu avais voulu me tuer au chalet de mon grand-père, je t'aurais prêté main-forte" (Ibid., 38). La sexualité chez Nelly exclut donc tout rapport d'échange basé sur "[la] pris[e] en compte [d]es désirs différents de deux corps, de deux sexes" (Dardigna, 1981: 86) et impose une opposition sujet-objet dans une dynamique de dépersonnalisation du féminin afin de faire régner la domination du masculin. 
Nelly dévoile, toutefois, les dessous de cette aliénation, qui la dépersonnalise même dans sa vie intime, en montrant qu'elle est le résultat d'un discours répété tel un leitmotiv dans l'univers médiatique et commercial, dictant à la femme le comportement à suivre pour préserver l'image de l'idéal féminin:

Par crainte que tu te lasses je résistais un peu [...] Dans les magazines de mode, on dit que les femmes ne doivent pas obéir au moindre début d'érection de leurs hommes au nom de leur désir, on dit aussi que les femmes doivent représenter pour eux un défi en opposant une volonté propre faite de retenue et ce, pendant la première phase de la relation [...]. (Arcan, 2004: 39)

De ce fait, l'inconscient collectif et notamment féminin est soumis aux "miroirs déformants des rapports sociaux de sexe, miroirs travaillés et retravaillés par le commerce, les rapports de pouvoir [...] (Marzano, 2002: 138).

Cependant, la narratrice de Folle est très consciente de sa dépersonnalisation et elle y participe activement. Elle nous livre, par exemple, ses impressions quand son amant a essayé de l'initier à la cyberpornographie:

Pendant un temps, tu as voulu prendre les choses en main en m'initiant à la cyberporno. [...] Pendant cette période, j'ai commencé à paniquer pour de bon parce que j'étais prête à tout pour te plaire et même à devenir client. J'ai eu peur que la collaboration ne soit l'unique façon de m'en sortir auprès de toi. (Arcan, 2004: 99).

Dans le passage ci-dessus, la narratrice montre qu'elle est prête à devenir un simple “client" et à se transformer en un objet dévitalisé pour pouvoir garder son amour. Le vocable “collaborer" souligne, par ailleurs, l'aspect artificiel et superficiel de la participation de Nelly dans la relation sexuelle, elle n'est pas donc au centre du plaisir mais plutôt un agent de sa mécanisation. En outre, la narratrice révèle qu'elle peut se montrer carrément absente dans sa sexualité en se détachant de son propre corps:

Après avoir regardé les photos, on a regardé les séquences vidéo où elle sortait ses petits seins d'un soutien-gorge rouge avant de tirer sa petite culotte blanche sur le côté et de s'enfoncer un doigt dans la chatte en ouvrant la bouche [...]. Là tu as baissé mon pantalon pour tirer ma petite culotte sur le côté et me prendre par-derrière. [...] Dans un geste ultime de retrait, j'ai remis mon sexe à Jasmine, j'ai baissé les bras et je n'ai plus bougé. (Ibid.: 103).

La narratrice décide, donc, de quitter son corps et de s'effacer pendant l'acte sexuel pour donner l'occasion à son amant de lui substituer l'image d'une autre (Jasmine), suscitant davantage son désir, car ce qui importe plus dans l'imaginaire stéréotypé (homme actif/ femme passive) c'est exclusivement le plaisir de l'homme et non pas celui de la femme. Le corps de Nelly devient dans ce sens un simple objet matériel au service du plaisir de son 
amant, il est donc une enveloppe charnelle dont elle peut se détacher: « Je laisse mon corps à l'autre, pas de problème; moi, je suis ailleurs" (Huston, 2011: 9) et fait engendrer ainsi un “moi-désincarné” (Laing, 1979: 90). Nelly franchit ainsi la deuxième phase de l'aliénation, celle du "dessaisissement" car dépossédée de son corps, ce dernier lui devient étranger et acquiert sa propre autonomie.

La sexualité, chez Nelly, agit donc comme dépersonnalisation et effacement identitaire: “en m'effaçant j'ai aussi perdu la mémoire des gestes à faire devant un homme [...] d'un seul coup je ne savais plus comment bouger ni gémir et toute la pièce s'est engourdie"; le corps de la narratrice lui est ainsi devenu étranger et incontrôlable "en sortant de mon corps c'est mon corps qui a pris le dessus" (Arcan, 2004: 104). La narratrice est ainsi aliénée au seul commandement de son homme: «Tes yeux se clouaient dans les miens et tu me serrais la gorge avec une main où passait ta force de chef en face d'une décision à prendre» (Ibid., 29), sa dépendance est alors remise en question: “tu m'as ramenée au degré zéro de l'autonomie" (Ibid., 39), face à son amant, l'aliénation devient sa seule réalité car "l'aliénation ne se confond pas avec dépendance. Elle se produit quand la dépendance tourne mal" (Gounelle et Reymond, 2004: 52).

C'est donc au nom de l'amour que Nelly abandonne son corps à son amant dans un geste d'immolation de soi où son propre plaisir est toujours absent: "je t'ai laissé me tirer les cheveux et me cracher dans la bouche [...] Avec toi j'ai connu des moments d'engourdissement que connaissent ceux qui sentent venir la mort" (Arcan, 2004: 38). La sexualité chez Nelly est par voie de conséquence un espace de soumission mais surtout d'absence et de paralysie face à l'autre, et non pas un échange de plaisir subjectivant les deux partenaires.

Toutefois, chez Arcan écrire sur le sexe ne se fait pas dans un but d'écriture érotique mais c'est surtout un moyen de démasquer des réalités dont le regard individuel et même social se détournent souvent, elle l'explique à juste titre:

Je ne suis pas là pour flatter les lecteurs dans le sens du poil, surtout pas les hommes. Je trouve qu'on vit dans une société où les images confrontent très peu, elles vont dans le sens de l'idéal, du rêve qu'on veut vendre, du succès, du bonheur. Moi, quand j'écris, j'essaie d'aller à l'envers de ça. Je pense qu'on ne peut avoir une réflexion sur le monde dans la complaisance, qui va mener à une sorte d'assentiment du lecteur. J'aime plutôt lui montrer ce qu'il ne veut pas voir. Jamais je ne vais accepter de générer par mon écriture le plaisir sexuel. C'est vraiment important pour moi, parce qu'on baigne là-dedans constamment. (Arcan citée in Guy, 26 août 2007).

La sexualité dans Folle est ainsi présentée comme un espace de dépersonnalisation et d'absence dont souffre l'auteure-narratrice, une situation qu'elle désapprouve mais à laquelle elle se soumit en étouffant en elle un cri de désespoir. 


\section{Conclusion}

L'identité queer dans Folle se manifeste ainsi au travers d'une subversion des genres, une subversion menée à la fois dans deux sens: d'une part en remettant en question la hiérarchisation socioculturelle basée sur la dominance masculine, et d'autre part en exprimant une démission face au genre féminin. En effet, malgré les efforts du féminisme à réhabiliter le statut et les droits de la femme au sein de la société, Arcan attire l'attention sur le fait qu'un tel combat politique pourrait être stérile face au genre féminin psychiquement aliéné et autoaliéné par les discours normalisés comme elle le déclare: "La femme possède une valeur intrinsèque par sa beauté et sa jeunesse. Toute la société nous ancre ce message-là. Je conteste cette dictature, tout en acceptant de jouer son jeu. Partir en guerre n'est pas mon rôle. Le mien consiste à devenir miroir" (Arcan, citée in L'aliénation de la femme). Selon Arcan, le consentement de la femme répond à l'obsession du genre féminin excluant de ce fait l'influence du patriarcat: "parce que c'est vraiment entre elles que ça se passe, je trouve. Les femmes vont beaucoup trop loin dans le consentement à la chirurgie pour que l'on puisse dire que ce sont les hommes qui veulent ça" (Arcan, cité in Guy, op.cit .). Arcan insiste donc sur le fait que "les femmes sont responsables de leur aliénation. Pour nous, les hommes ne sont que des instruments de mesure. Pour savoir laquelle est la plus belle" (Ibid.).

En somme, l'auteure-narratrice de Folle ne se révolte pas seulement contre les facteurs socioculturels qui l'aliènent mais elle s'insurge également et surtout contre sa propre nature de femme ayant tendance à s'autoaliéner. Elle adopte alors une attitude passive pour marquer son absence face à son amant et aussi face à elle-même; cette passivité/négativité est à la fois une "non productivité" sur le plan personnel et social. Elle commence d'abord par une déconstruction de l'image de soi et par extension du genre féminin, et cela en se dévalorisant face au regard de l'Autre, et en manifestant également un désir de changement de genre en voulant passer vers l'autre rive; et enfin en refusant catégoriquement une projection dans le futur et cela en programmant un suicide précoce qui lui permettra de quitter définitivement la condition du genre féminin. Nous estimons donc que c'est à travers cette révolte manifestée contre d'une part les rôles octroyés aux deux genres au sein de sa société et d'autre part contre le consentement du genre féminin, que se manifeste la construction d'une identité queer chez l'auteure-narratrice de Folle.

Cette identité queer qui transparaît ainsi au travers de la négation de soi et de la subversion des genres est avant tout une manifestation de l'individualisme extrême au cœur de son désir et de son besoin, mais Arcan a voulu l'élever au rang du collectif car, comme on l'a vu plus haut, "le personnel est politique". En effet, l'écriture autofictionnelle qui est une écriture altruiste et non pas narcissique, comme le défend son inventeur Doubrovsky, a permis à l'auteure de Folle de ranger son combat dans un mouvement social, collectif, pluriel et solidaire. Dans un travail ultérieur, il serait intéressant d'étendre l'analyse de ces stratégies de subversion du genre sur un corpus plus 
large, et toujours bien contextualisé, afin de dévoiler le pouvoir de la construction d'une identité queer sur la construction d'une identité féministe collective et contemporaine.

\section{Références bibliographiques}

ArCan, Nelly. 2001. Putain. Paris, Seuil

ArCan, Nelly. 2004. Folle. Paris, Seuil.

ArCAN, Nelly. 2004. La belle et le dragon (28 aout). [consulté le 17-12-2018] <http://www. ledevoir.com/culture/livres/62384/nelly-arcan-la-belle-et-le-dragon OFFEN>, Karen. Flux et éruptions: réflexions sur l'écriture d'une histoire comparée des féminismes européens 1700-1950 in COVA, Anne (dir). 2009. Histoire comparée des femmes. Paris, Publisud.

Banderole du groupe Psychanalyse et politique, manifestation du 6 octobre 1979.

Beauvoir, Simone (de) (1976 [1949]). Le deuxième sexe I. Paris, Gallimard.

Butler, Judith. 2005. Trouble dans le genre:le féminisme et la subversion de l'identité, traduit de l'anglais par Cynthia Kraus. Paris, La Découverte.

Cornaton, Michel. 1990. Pouvoir et sexualité dans le roman africain. Paris, L'Harmattan.

"Nelly Arcan -La belle et le dragon" 28 août 2004. Art. [consulté le 17-12-2012]: <http:// www.ledevoir.com/culture/livres/62384/nelly-arcan-la-belle-et-le-dragon>

DARDigna, Anne-Marie. 1981. "Les châteaux d'Éros ou l'infortune du sexe des femmes" in Les Bulletins du GRIF, $\mathrm{n}^{\circ} 4$.

DerridA, Jacques. 1972. La dissémination. Paris, Seuil.

Dorlin, Elsa. 2008. Sexe, genre et sexualités. Paris, PUF.

Définitions sur la diversité sexuelle et de genre. 2016. [consulté le 12.11.2018]: <http://interligne.co/faq/que-signifie-le-terme-allosexuel-queer/>

Dupont/DelPhy, Christine.1970. “L'ennemi principal” in Partisans 61-64.

FEDIDA, Pierre.1978. L'absence. Paris, Gallimard.

FIRESTONE, Shulamith. 1972. La dialectique du sexe. Traduit de l'américain par Sylvia Gleadow. Paris, Stock.

Gounelle, André et Reymond Bernard. 2004. En chemin avec Paul Tillich. Munster, Lit Verlag.

Guy, Chantal. 26 août 2007. Nelly Arcan: l'amour au temps du collagène. La Presse. [consulté le 10.10.2018]: <https://www.lapresse.ca/arts/livres/entrevues/200909/25/01-905440-nelly-arcan-lamour-au-temps-du-collagene.php>.

Huston, Nancy. 2011.“Arcan, philosophe” in ARCAN, Nelly. Burqa de chair. Paris, Seuil. 
Anales de Filología Francesa, n. ${ }^{\circ}$ 27, 2019

IDENTITÉ QUEER: ABSENCE ET NÉGATION DE SOI DANS FolLE DE Nelly ARCAN

LACAN, Jacques.1973. Le séminaire. Les quatre concepts fondamentaux de la psychanalyse. Livre XI. Paris, Seuil.

LACAN, Jacques. 1957-1998. Les formations de l'inconscient, Le séminaire, livre V. Paris, Seuil.

LAIN, Ronald David.1979. Le moi divisé, de la santé mentale à la folie. Traduit de l'anglais par C.Elsen. Paris, Stock.

L'aliénation de la femme [consulté le 15.02.2018]:<http://www.nellyarcan.com/pages/theme-alienation.php>.

Marzano, Michela. 2002. Penser le corps. Paris, PUF.

Marzano, Michela. 2013. La philosophie du corps. Paris, PUF, coll. “Que sais-je?”.

Millet, Chatherine. 2002. La vie sexuelle de Catherine M. Paris, Points.

Minne, Samuel. 2009. “Queer Readings”: lectures de la différence. [consulté le 13.10.2018]: $<$ http://www.fabula.org/atelier.php?Queer_readings>.

Pico, Francois. 2010. "Vous avez dit dit queer? La question de l'identité et du féminisme". In Réfraction24. [consulté le 30.10.2018] <https://refractions.plusloin.org/IMG/pdf/2401.pdf>

Witting, Monique.1980. On ne naît pas femme" in Questions féministes n 8, 56-72. 